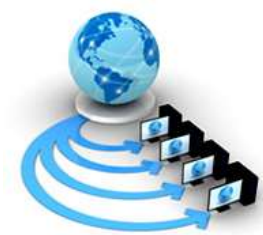

Volume 9, No. 6, November-December 2018

\title{
GEO-FENCING APPLICATION FOR NATURAL DISASTER AND STUDENT TRACKER
}

\author{
Mr. Nishant. G. Hulwan \\ Computer Department \\ MGM's College of Engineering and Technology \\ Navi Mumbai, India.
}

\author{
Prof. Sachin chavan \\ Computer Department \\ MGM's College of Engineering and Technology \\ Navi Mumbai, India
}

\begin{abstract}
Communication during College entry and exit is very crucial for both college and the department with Disaster for the user help. Student and faculty never come before time which can be very crucial when needed. This system can be used to function in case of crisis within the College for either student came or not in the college premises. Emergencies which include Enter in the college premises, Exit of the student from the college, And notification to the admin pane for that the student enter or not in the college premises. In this thesis paper, our objective is to present Android Based Artificial Intelligence Support for Emergency Information bot for the college staff and students. To utilize GPS Tracker, this enables Android phones GPS tracker at College premises times over any Internet Connection or Wi-Fi.
\end{abstract}

Keywords :Google Fence, Support Vector Machine, Attendance, Disaster, Android.

\section{INTRODUCTION}

College, School or human-induced has hit the country in the past and no wonder hit in future too. A disaster is a natural event or human-made event that can negatively affect humanity. Thus, to prevent this calamity we should take proper precautions, and everybody should react in patients so that we can save more life's and properties and, because of this a functional-oriented and action-oriented is going to help people to communicate in such disaster. Students come into the college premises is essential. Anybody who is in an emergency is calling the College department. Here we are going to use Location Co-ordinates which works as a sending request through the use of GPS tracker. This system is going to work as a Client-Server system, where the server is going to respond to the request of the client. This System implemented as an Android-Based Java Application Daniel C. Sucgang focused on this topic [1]. Different emergency handling services are present until now to lend a hand the various types of emergencies faced in the present. Entry of students in the college is the critical factor one of the organizations that help, scattered different College issues. College at times hit, wherein the communication is very hard, and longer available when utmost it is needed. Recent Colleges like entering of the students in Samar have caused too much Disturbing and massive loss of Database for the admin panel to manage, lack of alternative communication solutions at rescue places. The mobile phone application permits users to get tour guidance information that they can access anywhere they want. The increase in the growth of mobile phones has led to a resurgence for mobile services. Go anywhere applications support a wide array of social, financial, and enterprise services for any user with a cellular data plan.

Mobile devices are progressively becoming a part of humanity, as the most effective and convenient communication tools not bounded by name and place. This research is intended to construct a system that provides time to time entrance to general, pertinent, and reliable information that is critical to humanitarian operations, as well as operations management, and this also includes the automatic update of news online, especially on social media. The main objectives of this system is to execute the artificial intelligence with the help of Global positioning system tracker for android device; it also provides reliable and comprehensive support for the community that is in need; therefore to help various organizations leading to disaster and reduce risk management we use the GPS tracker feature of the android device. This proposed study executes through mobile phones operated by the android users. Any user who has connectivity to the internet via WIFI can benefit from this system.

\section{EXISTING SYSTEM}

The commercially available cell phone geofencing products reviewed in this document relies on GPS speed; geo-location or new hardware devices can disable the usage of the phone while driving Survey of Existing technologies [2]. When applying the geofencing products for the NT project, however, the products that use the GPS speed for driver distraction prevention won't work because they falsely trigger when used on a transit vehicle, which are times when the application should not be disabled. Therefore, the existing geofencing technologies based on detecting GPS speed or locations (either software only or software and hardware solutions) usually fitted into the NT project's needs.

However, the products that use an additional in-vehicle device for distracted driving prevention can conditionally solve the problem for cars that have the equipment installed. The requirement of connecting an extra device is more costly than not joining a machine, and therefore is a less desirable solution for the geofencing needs of the project. For the NT project, we developed a geofencing function as part of the mobile software so that the use of the application can be disabled whenever the user is detected to be driving based on the GPS tracks and itineraries. 
2.1 Existing System (Geo Fence Application)

(1) Static Virtual-notification: This application works on the geographical position of a mobile user concerning a fixed area. For Example, the mobile user gets a notification when he/she entered into geo-fence, as the student comes into the school campus.

(2) Dynamic Virtual-notification: This application is rooted in the geographical location of a mobile user concerning a changing data stream. For example, the "open parking space" mobile user gets notified that who happen to be driving nearby.

(3) Peer-to-Peer virtual-notification: This application works on the geographical location of a mobile user concerning other users. For example, a person may go through notification of nearby friends on a mobile app like Facebook, Instagram or Foursquare proposed by Sachin W. Rahate [3]. The geo-contextual trigger fires correctly function of: Location Accuracy - The device location must be correctly identified to a geo-fence for the action to occur appropriately (i.e., the right user receives a notification).

A. Tracking Rate -Location is calculated by cadence which provides a location update to the server, where the device is to get the measure against available events (with associated actions) actions.

B. Device Speed - The speed of the device determines the time within which, the invention is necessary to provide a location and update to be estimate against single events (with associated actions).

C. Device Route - Device route is a device that takes across a virtual fenced area which affects the period within which location update must occur. For example, the time span is shorter if the method follows the edge of a circular geo-fence rather than passing through the center.

D. Size of Geo-Fence - A larger Geo-fence provides a longer time for the update of a location unless the device tracker itself track skirts the edge of the Geo-fence.

\section{- How fleet management is used in Geo-Fencing using IoT?}

Fleet management can be used in different ways in IoT:

(1) Planning trips \& Scheduling trips: Fleet management uses planning trips and Scheduling trips in such a way that owners can plan their own trips in a specific manner using Geo tracking and communication with the help of IoT.

(2) Fleet management- When a truck driver diverts from his route, the dispatcher get an alert. This is used as fleet management using Geo-Fencing [4].

(3) Maintenance: There are hundreds of vehicles operating on fleet management, so it is crucial to have conservation of these vehicles and the consequences of inappropriate actions.

\section{Android}

Android is a mobile operating system and another open source Linux-based Operating System for mobile phones such as smartphones and tablet computers. Android led by Google, and other companies. Android has given a rare approach to application development for mobile devices, for example, A developer develops an application for Android, he uses different programming languages to build the application and he uses various tools to create it and once completed their claims should be able to run on different devices supported by Android proposed by Przemyslaw Gilskil [5].

Android applications developed in different languages like Java language, $\mathrm{C}$ language, $\mathrm{C}++$ language, Python language, $\mathrm{C} \#$ language using the Android SDK (Software Development Kit), Eclipse Photon, and different tools. Once the application finished, the forms can be used quickly and sold out either through a store such as Google Play, Slide ME, Opera Mobile Store, Mobango, F-droid, and the Amazon App store. There are millions of Android mobile device usages in more than 190 countries around the world. It is mobile platform independent, fast \& most installed app. Each day more than 1 million new Android devices are activated across the globe.

\subsection{Android IDEs}

- In the modern day, the technology is everywhere, and there are so many sophisticated Technologies available to develop android applications, the familiar techniques, which are predominantly using tools as follows

- Android studio

- Eclipse IDE Photon

- Fabric

- Flow Up

- Game maker

- Geny motion

\section{Implementation Methodology}

Artificial Intelligence spotlights the creation of the smart machines that work and responds like humans several other activities of computers with Artificial Intelligence is designed to include Speech Recognition, Learning, Planning, and Problem-solving as proposed by Mrs. Rekha Purohit1 [6]. Machine learning is another fundamental element of AI. Learning without any supervision requires the ability to recognize patterns in streams of inputs while learning with sufficient guidance involves classification and statistical regressions. Classification decides on the category an object belongs to the regression model and deals with obtaining a set of numerical input or output. For example, let's say we are discovering functions and enabling the production of the appropriate outputs from respective data. One thing we can do is use Machine perception that deals with the capability to utilize sensory data to figure out the diverse aspects of the world. Computer vision is still the power to analyze visual data with fewer problems such as facial object and speech recognition. Artificial Intelligence can take more stressful, complex works than humans do, this system can make the task faster than a human can so it is most favorably to discover many unexplored things than humans. It can restore human resources in every field, which results in human beings left abandoned. Replacing human beings with Artificial intelligence bots in the area is not going to work out. Artificial Intelligence machines do not provide the needs and substitute for caring things that human service. The major disadvantage of Artificial Intelligence machines is that they cannot be 'human' they can only think but they can't feel. Artificial intelligence is used in various applications today they are mostly used to send rovers to Mars and different planets in 
our solar system. Artificial Intelligence will be very helpful to humans.

\subsection{Algorithm Used}

"Support Vector Machine" (SVM) is a supervised machine learning algorithm it is a series of data for both classification or regression challenges in the geo-fencing application for the disaster and college application. Moreover, it is applicable in classification problems for the user enters the location, so that system after that application algorithm works stated by Ithipan Methasate [7]. In this technique, we plot each data item as a point in $\mathrm{n}$-dimensional space (where $\mathrm{n}$ is some features) with the value of each function being the value of a particular coordinate of the data. Then, we perform classification by finding the hyperplane that distinguishes the two classes very well.

$A$. How does this method work?

In the above method, we got accustomed to the process of segregating the two classes with a Question. Now the most primary thing is "How can we identify the right Authentication question?" It's not that hard as you probably think.

Let's understand:

- Identify the right Authentication user (Scenario-1): Here, we have three Questions (A, B and C). Now, identify the right Question to classify star and circle.

We need to remember a thumb rule to identify the right Question for the Answer: "Select the random number question which segregates the classes better." In this scenario, Question "B" has excellently performed this job.

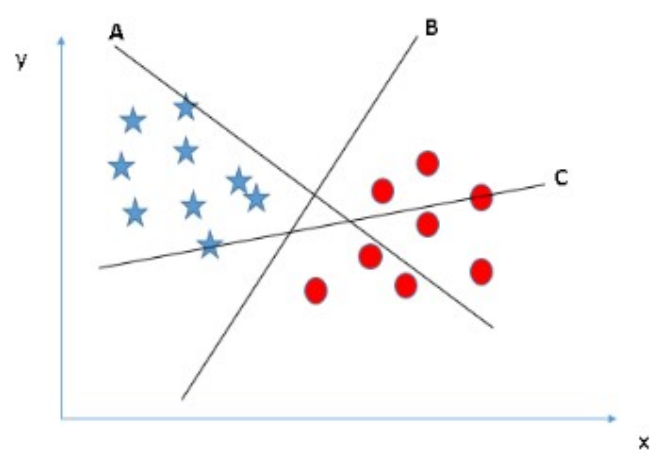

\section{PROPOSED SYSTEM}

Geo-fencing is a location-based service in which an app or other software uses GPS, and We can also use Wi-Fi or cellular data here to trigger a pre-programmed action when a mobile device tag enters or leaves a virtual boundary set up around a geographical location known as a virtual fence. Depending on how a virtual-fence can build it can prompt mobile push notifications, trigger text messages or alerts. Moreover, send targeted Disaster information and college department on mobile, allow tracking on vehicle fleets, and disable specific technology or delivery location-based marketing data Some Geo-fences are used to monitor activity in secure areas, allowing management people to see alerts when anyone enters or exits a particular location. People who are working in the business can also use virtual fencing to keep a check on their employees and their respective field, and they can also keep track on electronic time cards and pay attention to company properly. This location allows management to see alert flags when anyone is leaving the office or entering the office area.

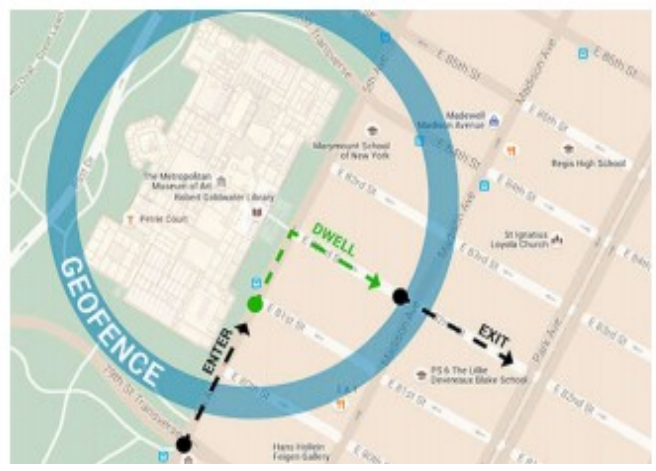

Geo Fencing Action Diagram

\section{How Geo-fencing works?}

To make use of Geo-fencing, an administrator or developer must first establish a virtual boundary around a specified location in GPS software. There is the simplest way to draw a circle around 100 feet a place on Google maps, as specified using APIs while developing a mobile app. This Geo-fence will then trigger a response when an authorized device enters or exits that area, as determined by the administrator or developer proposed by Ankita Sakore [8]. A virtual fence is the most commonly defined within a code of a mobile application especially when users need to opt-in to locate service for the virtual wall to work correctly. If you go to a College special event, they might have an app made by the students there which you can download. After downloading it delivers information about the Disaster or college information and, this might draw a virtual-fence around its outlets to trigger mobile alerts for customers who have downloaded the User's mobile app. In these cases, a Geo-fence that is managed by the User and it is programmed into the application so that users can opt to decline location access for the app. A Geo-fence can also be set up by end-users using virtual-fencing capabilities in their mobile apps. Virtual-fence can also be set up by people who use the actual product using virtual-fencing capabilities in their mobile apps. There are different applications such as, Android reminders which allow you to select an address or location where you want to stimulate a specific alert or push notifications. This term is called an "if this case, then do these" command, where an app is programmed to trigger or generate an action based on another action. For example, "If I am five feet from my front door, then turn on my lights." Alternatively, you might ask a reminder app to send you an alert or trigger once you reach a specific location.

\subsection{How to use virtual-fence}

The system using virtual fencing is possible to deliver the disastrous information to the user who has just entered the fence. In this research, we implement Geo-fencing with the Core Location framework of Android. This framework gives a detection of the entries and exits of the user with the observation of a specific geographic region. The geographic 
region area which is defined by a circle within a specified radius around is known as the point on Earth. Every-time the user who uses the application crosses the boundary of the region, and then the system generates an event for the form proposed by Komal Wagh [9]. This application sanctions the notification of the disaster statistical data. It is possible, by using the observations of a geographical and demographical area; it is possible to detect user behavior in the same manner as the definition of virtual-fencing.

Moreover, the system does not report an event until the user enters into the region further from the boundary plus a system-defined cushion distance. This cushion value is not allowing the system to generate numerous occasions while the user is traveling near to the edge. The cushion distance identifies the hardware and the location technologies that are currently present.

\section{LITERATURE REVIEW}

This part of the thesis paper we have tackled all the reviewed references and works related to the system developed in the above module. In one, previous dissertations, journals, and practices associated with this paper will be used to give the reader a better understanding of what the researcher is trying to demonstrate.

\section{(1) Related Literature}

The review of related literature conducted by individual professionals, authors, graduate and undergraduate thesis writers on systems related to the study has profited the researcher with information that serves as a basis for the development of the proposed method. It will also rationalize the fact the proposed system is possible to develop. On maps for plotting crimes, crime analysis has relied. Planning crimes after they occur is a static process of historical data collection and reporting wherein data might not be as planned as the schedule for many days, weeks, or months after the criminal event. SMART (Spatial Management, Analysis, and Resource Tracking) mapping in law enforcement settings means integrating geographic information systems with dynamic location acquisition technology where near-real-time data collection and analysis are possible. This paper explores several possibilities for dynamic near-real-time mapping applications for law enforcement. The examination is potential uses for small hand-held field equipment to plot "hot spots network," boundaries, and another geographic status, and for largely automated vehicle location and artificial intelligence-guided emergency services dispatch (Sorensen, Severin L., SPARTA Consulting Corporation, "SMART Spatial Management, Analysis and Resource Tracking, 2008”). Mobile Emergency Response Application uses Geolocation for Command Centers. An application used to request a quick response calling people for help in many places. This Geolocation was used to observe the location of the user and information of a user (Jethro B. de Guzman, Ritz Carlo C. de Guzman, and Engr. Remedios G. Ado, "Mobile Emergency Response Application Using Geolocation for Command Centers," 2014).

A novel Smartphone-based communication framework. It uses specific machine learning techniques that sharply process sensor readings into useful information for the crisis responders. Core to the structure is a content-based publishes-subscribe mechanism that allows flexible sharing of sensor data and computation results. We have also to evaluate a preliminary implementation of the platform, involving a Smartphone application that reads and shares mobile phone sensor data for activity recognition (Radiant, Jaziar et al., "Smart Sensing Platform for Emergency Management."

An expert system is a system where a computer program is coded to simulate the knowledge and behavior of an individual or an organization which is expert in some particular field. Usually, all expert systems contain a knowledge base which is accessible by a set of rules depending on specific situations. An expert system (ES) is knowledge-based which can be updated and extended. This system employs knowledge about its application domain and uses inference (reasoning) procedure to solve problems that would otherwise require human competence or expertise proposed by KP tripathi [10]. These include areas such as a high-risk acknowledgment or credit decisions or marketing decision making and manufacturing decisions. The general areas of ES applications where ES can be applied it includes this module we are going to study on the different classification, diagnosis, monitoring, process control, design, scheduling and planning, and generation of options that contain a large amount of information. They are not capable of learning from mistakes. Thus it can't creatively come with new solutions for issues, and lastly, it is hard to achieve the exact knowledge of an expert.

\section{System Architecture Diagram}

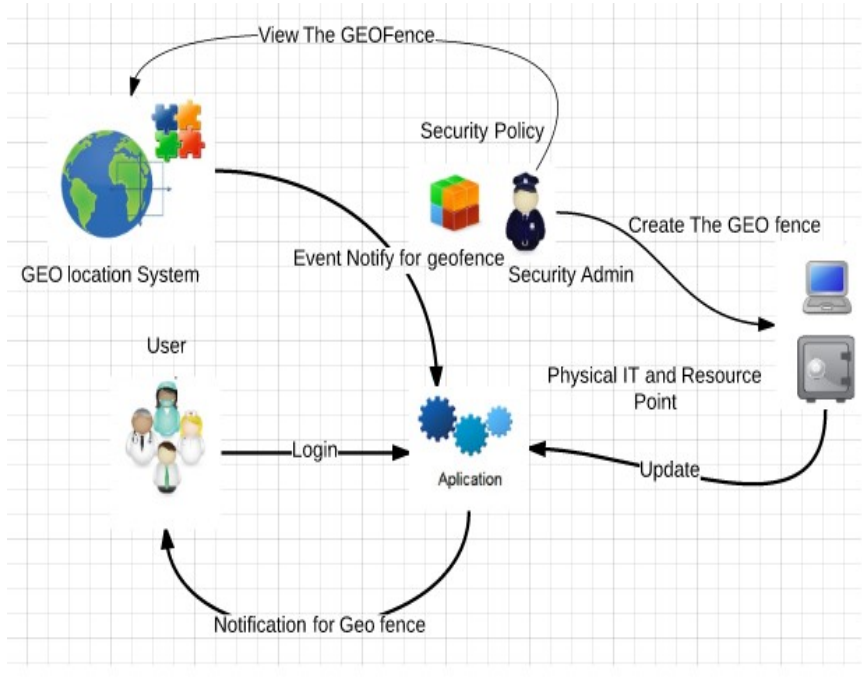

\section{ADVANTAGES}

1. The Proposed system provides a feature where you can check your daily Notification.

2. You can manage your Travel Path

3. No need to remember the disaster occurring area while traveling.

4. Faculty and parent get the notification about the students via android application.

5. Verifying your GPS becomes easier. 


\section{APPLICATIONS}

- Geo-fencing, used with child safety, if child enter or leaves designated area then parent can get notification.

- In shopping tracker, user can get alert about shopping reminder for example shopping list notification when user will be near to shopping location.

- In Marine safety service, if ship will move towards prohibited area or international border then before some predefined distance notification will be send to ship.

- Human resource management- An employee smart card or GPS tracking device will send an alert to security if an employee attempts to enter an unauthorized geofence/area. -Compliance management- Network logs record all geo-fence crossings to document when the proper use of devices and their compliance with established rules.

- Marketing- A restaurant can send text message with the day's specials to customer, when the customer enters a defined geographical area or geo-fence. -Asset management- An RFID tag on a pallet can send an alert or notification, if the pallet is removed from the warehouse without authorization.

- Law enforcement- An ankle bracelet can send notification or alert authorities if an individual under house arrest leaves the premises

\section{CONCLUSION}

This research gives overall engineering that will help students and staff in various college information systems for the entering in college premises and leaving from the college premises using their Android Smartphone and with the help of Virtual Fencing for their information. It is also a help to the students of College departments that lack communication of victims to the authority during College informatics.

\section{REFERENCES}

[1] Daniel C. Sucgang, Jennifer T. Villanueva, Nea Cris C. Macawile, Richard Cedric Antivo, Irish Paulo R. Tipay," An Android Based Artificial Intelligence Emergency CP(Crime Prevention) Response System Using GPS Tracker," International Journal of conceptions on computing and Information technology, 2015.

[2] A Survey of Existing Technologies, Applications, Products, and Services for Geofencing, California PATH Program University of California at Berkeley,2010.

[3] Sachin W. Rahate, Dr. M.Z. Shaikh," Geo-fencing Infrastructure: Location Based Service," International Research Journal of Engineering and Technology, 2016.

[4] Fleet Management using Geo Fencing - An IoT Use Case, 2016.

[5] Przemyslaw Gilski1, Jacek Stefanski, Android OS: A Review, TEM Journal, 2015.

[6] Mrs.Rekha Purohit1 Mrs. Snehlata Vyas2 Dr. Prabhat Mathur, "AI and its Application: Sixth Sense Technology" International Journal of Emerging Trends \& Technology in Computer Science (IJETTCS), 2013.

[7] Ithipan Methasate Thanaruk Theeramunkong

a. Experiments on kernel tree support vector machines for text categorization", PAKDD'07 Proceedings of the 11th Pacific-Asia conference on advances in knowledge discovery and data mining, 2017.

[8] Ankita Sakore, Dhirajkumar Patil, Amol Thukrul, Babita Bhagat," Virtual-fencing for a Disaster Information using Android," International Journal Of Innovative Research Explorer,2018.

[9] Komal Wagh1, Shreya Thakur2, Saraswati Yadav3, Assistant Prof.Shivsager Gondil," Disaster Information System Using Geofence," International Journal of Recent Trends in Engineering \& Research, 2018.

[10] K P Tripathi," A Review on Knowledge-based Expert System: Concept and Architecture" IJCA Special Issue on "Artificial Intelligence Techniques - Novel Approaches \& Practical Applications, 2011. 